\title{
Implementation of a Clinical Decision Support System for Precision Oncology across an Academic Network
}

David Tamborero ( $\sim$ david.tamborero@gmail.com )

Karolinska Institutet

Rodgrigo Dienstmann

Vall d'Hebron

Maan Rachid

Karolinska Institutet

Jorrit Boekel

Karolinska Institutet

Adria Lopez-Fernandez

Vall d'Hebron Institute of Oncology

Irene Braña

Vall d'Hebron Institute of Oncology

Luigi De Petris

Karolinska Institutet

Jeffrey Yachnin

Karolinska Institutet

Richard Baird

Cancer Research UK

Christophe Massard

Gustave Roussy Cancer Campus

Frans Opdam

The Netherlands Cancer Institute

Richard Schlenk

German Cancer Research Center

Claudio Vernieri

Istituto Nazionale dei Tumori

Michele Masucci

Karolinska Institutet

Xenia Villalobos

Vall d'Hebron Institute of Oncology

Elena Chavarria 
Vall d'Hebron Institute of Oncology

\section{Judith Balmaña}

Vall d'Hebron Institute of Oncology

\section{Giovanni Apolone}

Istituto Nazionale dei Tumori

\section{Carlos Caldas}

Cancer Research UK Cambridge Institute, University of Cambridge, Cambridge https://orcid.org/00000003-3547-1489

\section{Jonas Bergh}

Karolinska University Hospital and Karolinska Institutet

\section{Ingemar Ernberg}

Karolinska Institutet

\section{Stefan Fröhling}

National Center for Tumor Diseases https://orcid.org/0000-0001-7907-4595

\section{Elena Garralda}

START Madrid, Hospital Universitario Madrid

\section{Claes Karlsson}

Karolinska Institutet

\section{Josep Tabernero}

Vall d'Hebron Institute of Oncology (VHIO) https://orcid.org/0000-0002-2495-8139

\section{Emile Voest}

Netherlands Cancer Institute https://orcid.org/0000-0001-8249-9586

\section{Jordi Rodon}

Vall d'Hebron University Hospital and Institute of Oncology (VHIO)

\section{Janne Lehtiö}

Karolinska Institutet https://orcid.org/0000-0002-8100-9562

\section{Article}

Keywords: clinical decision support, precision oncology, Cancer Core Europe network

Posted Date: April 21st, 2021

DOl: https://doi.org/10.21203/rs.3.rs-401975/v1

License: (c) (i) This work is licensed under a Creative Commons Attribution 4.0 International License. Read Full License

Version of Record: A version of this preprint was published at Nature Cancer on February 24th, 2022. See the published version at https://doi.org/10.1038/s43018-022-00332-x. 


\section{Abstract}

There is a growing need for systems that efficiently support the work of medical teams at the precision oncology point-of-care. Here we present the implementation of the Molecular Tumor Board Portal (MTBP), an academic clinical decision support system that creates a unified legal, scientific and technological platform to share and harness next-generation sequencing data across the Cancer Core Europe network. Automating the interpretation and reporting of sequencing results decreased drastically the need for manual procedures that are time consuming and prone to errors. In addition, the adoption of an expertagreed process to systematically link tumor molecular profiles with clinical actions promoted consistent decision-making and structured data capture across centers. Finally, the use of information-rich patient reports with interactive content facilitated collaborative discussion of complex cases during virtual molecular tumor board meetings. Overall, we believe that streamlined digital systems like the MTBP are crucial to better address the challenges brought by precision oncology and accelerate the use of emerging biomarkers.

\section{Introduction}

Next-generation sequencing (NGS) assays are a key component of the modern oncology workflow. Beyond on-label drug prescriptions, tumor sequencing results can guide clinical trial enrollment and identify investigational drug opportunities in individual patients. NGS data can also reveal other events of clinical relevance, such as germline pathogenic variants, pharmacogenomics findings and clonal hematopoiesis drivers, which should be recognized and acted upon. However, clinical interpretation of NGS results often relies on manual procedures, which poses considerable challenges to the medical teams undertaking this task. First, variant annotation benefits from numerous resources developed by medical, biological and bioinformatics domains that are not easy to integrate. Second, agreeing on annotation criteria and rules to prioritize actionable findings is critical for consistent clinical decisionmaking. And third, in the absence of on-label treatment options, patients must be matched with the specific portfolio of investigational therapies and clinical trials available in each hospital (or hospital network), which are subject to continuous changes. Failure to address these issues or the inability to perform them in a clinically acceptable time frame can impair the outcome of individual patients and precision cancer medicine initiatives.

Clinical decision support systems (CDSS) can tackle these challenges by implementing efficient data analysis and reporting processes. Several commercial CDSS software are currently available, but inhouse solutions are often used to better accommodate specific needs of each center. In fact, we believe that the capacity of academic institutions to develop custom CDSS accelerates the use of emerging biomarkers, promotes precision medicine across healthcare professionals and catalyzes clinical research. We have therefore developed the Molecular Tumor Board Portal (MTBP), a CDSS that creates a unified framework to interpret sequencing results across the seven comprehensive cancer centers that form Cancer Core Europe (CCE) 1 at present. Importantly, the portal is seamlessly integrated in the CCE clinical workflows and provides a single platform to distribute the results and support shared discussions at 
scale2. Seamless communication among clinical investigators is essential to leverage the collective expertise of the community in this era of rapidly changing precision oncology landscape. To our knowledge, this is an unprecedented effort for co-developing new anti-cancer drugs and biomarkers in Europe. Here, we describe our approach and discuss the results of using the MTBP in a consecutive cohort of 500 advanced solid tumors evaluated from January 2019 to January 2021 in the context of the Basket of Baskets (NCT03767075), an ongoing CCE multi-basket phase II clinical trial matching molecular biomarkers with immunotherapies and targeted drugs.

\section{Results}

\section{Functional interpretation of cancer variants}

Interpretation of NGS data first requires elucidating whether the variants observed in cancer genes can confer tumor-promoting traits, as not all of them have equal biological consequences. Besides the identification of the individual tumor genomic drivers, this analysis enables matching patients to biomarkers defined by functional criteria, such as 'activating' mutations in a given oncogene or 'loss-offunction' alterations in a given tumor suppressor. Of note, close to one third of the cancer biomarkers reported at present rely on interpreting the functional effect of variants found in drug targets (Supp Figure 1). This number will likely continue to grow as genes involved in more cellular processes, such as DNA damage repair, epigenetics, metabolism and immune-regulation, become actionable.

The MTBP interprets the functional relevance of cancer variants under an allele-centric perspective (Fig $1 \mathrm{~A})$. In other words, a given BRCA1 mutation known to disrupt the activity of the wild type allele will always be declared as functionally relevant (i.e. loss-of-function) regardless of context considerations such as the germline versus somatic origin of the variant, the status of the second allele and/or the cancer subtype in which it is observed, which are however contemplated in the actionability analysis (see next section). Multiple genomic knowledge resources can be integrated for a more comprehensive variants' functional annotation, but currently there are no well established guidelines on how to do it. Therefore, we agreed on criteria considered to provide strong or very strong supporting evidence (>90\% and $>99 \%$ of certainty, respectively) as extrapolated from previous work3 and based on three distinct sources of knowledge (Supp Figure 2A and 2B):

1. First, the MTBP inspects whether the gene variants observed in the tumor have an already wellreported effect. Note that different effect assertion types can be equally mapped to the contextagnostic notion of a variant being functionally relevant; for example, a given BRCA1 mutation can be considered to be a putative loss-of-function event both when reported to predispose to early breast/ovarian cancer or a known biomarker of PARP inhibitor response. Therefore, the MTBP queries a number of expert knowledge databases that continuously gather results of clinical, experimental and population genetic studies4-10 according to the standard procedure defined for each of their respective scopes (such as the pathogenicity classification of germline variants11), and assertions compatible with the functional relevance (or lack thereof) of the variant are then matched 
as appropriate (Supp Figure 2A). The aggregation of these knowledgebases, which are not often used in combination, enables a better use of international curation efforts (Supp Figure 2C).

2. Second, if no variant effect is reported, or the information is inconclusive and/or supported by weaker evidence (Supp Figure 2C), the portal evaluates whether bona fide biological assumptions (such as whether a given premature termination codon is likely to trigger nonsense-mediated decay) can be applied (Supp Figure 2D). These assumptions are largely based on accepted criteria to identify loss-of-function variants in Mendelian disease genes12. Of note, the MTBP refines the use of some of these criteria by leveraging the aggregated content of the aforementioned knowledgebases, which for example help to delineate protein regions that are critical for a given tumor suppressor function (Supp Figure 2D). This exemplifies the value of the MTBP for integrating the knowledge available in the community and developing ensemble bioinformatics models.

3. Third, if none of these bona fide assumptions can be applied or fulfilled, computational-based metrics are used as the lowest level of supporting evidence. For example, hotspots of somatic mutations observed across previously sequenced cancer cohorts point out mutated protein sites that are preferentially selected by tumors and thus relevant for the disease development in both oncogenes and tumor suppressors 13. In order to reduce the number of false positives, the MTBP uses methods that consider underlying genomic mutational processes to declare the accumulation of mutations of a given consequence type as statistically significant14,15. In addition, functional impact predictions can be also used to estimate whether other variants drive loss-of-function events. Among all the methods developed with that purpose, we decided to use deleteriousness score calculations 16 with stringent thresholds exhibiting a $90 \%$ of predictive value, as required for strong supporting criteria3, based on the results of our own benchmarking (Supp. Figure 2E).

Variants that cannot be classified as functionally relevant or functionally neutral according to any of the aforementioned criteria appear as of unknown functional significance in the MTBP reports. For the CCE prospective cohort presented here, composed of 500 solid tumors profiled by NGS panels (including up to 350 cancer genes; Table 1), the MTBP identified a median of 3 (interquartile range 2-4) functionally relevant mutations (single nucleotide changes and/or small indels) per tumor. Overall, and after excluding mutations assumed to be non-relevant (such as those that do not alter the protein sequence or are known polymorphisms), a total of $26 \%$ of the tumor mutations were classified as (putative) functionally relevant, while $9 \%$ were classified as (putative) neutral (Figure 1B). One fourth of these classifications were solely based on bioinformatic predictions, which as discussed is the lowest level of supporting evidence. Therefore, and even with the comprehensive functional annotation provided by the MTBP, most (65\%) of the tumor mutations observed in cancer genes remained of unknown functional significance (although this number largely varies across genes; Figure 1C). This illustrates our still limited ability for interpreting the biological relevance of the genomic alterations that occur in tumor cells. As drug prescriptions progressively move towards a more holistic consideration of the tumor genome (pathway and/or signature-centric), we stress the importance of using interpretation tools that are kept up-to-date with the knowledge provided by emerging capabilities, such as high throughput functional assays. 


\section{Clinical interpretation of cancer variants}

The final objective of the MTBP is to help translate NGS results into the most appropriate therapeutic decisions according to state-of-the-art knowledge. Genomic alterations that influence anticancer drug response (sensitivity or resistance) and that are of diagnostic or prognostic value are continuously reported in the literature and scientific venues. Several international initiatives gather this information in specific knowledgebases open for the access and feedback of the community6-8. However, these resources follow varying data models, and the accurate aggregation of their content requires an extensive harmonization of the lexicon, ontologies and genomic variant representation syntax employed by each. The MTBP implements this process with a semi-automatic pipeline that combines a number of bioinformatic mapping tools 17,18 and manually annotated dictionaries. The adoption of information exchange standards in the community is crucial to mitigate the need for these efforts and facilitate genomic knowledge sharing19-21.

The MTBP matches the cancer biomarkers aggregated across these knowledgebases with the variants observed in the tumor for (i) a specific nucleotide and/or protein amino acid change (such as 'KIT:p.D572A'); (ii) a variant category (such as 'EGFR in-frame deletions in exon 19'); or (iii) a functional entity (such as 'FLT3 oncogenic mutations', as guided by the MTBP functional interpretation) (Supp Figure $3 \mathrm{~A}$ ). However, as previously mentioned, variant actionability must also take into account additional tumor context considerations beyond the mere variant match, such as the concordance between the biomarker' and patient's cancer type (or a subtype thereof), the presence of co-occurring alterations that can influence the biomarker effect and the level of evidence that supports its clinical utility at present (Supp Figure 3B). The MTBP pipeline factors in these considerations as appropriate and automatically reports the results following the European Society for Medical Oncology (ESMO) Clinical Actionability of Molecular Targets (ESCAT) scale22, which is an extension of that previously presented by American expert associations23 (Supp Figure 3C).

The highest level of actionability corresponds to genomic alterations matching on-label prescriptions or clinical expert group recommendations, and thus ready to be used in routine clinical practice (ESCAT Level I; Figure 1D). However, in the context of CCE initiatives, we mostly profile tumors of patients without standard-of-care therapeutic options available. Consequently, investigational and off-label drug opportunities based on preliminary clinical (ESCAT Levels II and III) or even preclinical (ESCAT Level IV) evidence are also considered. In these cases, we prioritize the allocation of patients to clinical trials, and one key feature of the MTBP is therefore to detect cases eligible for those open for recruitment across the connected centers, as detailed next. Of note, the CCE network hosts the Basket of Baskets (BoB), an European multi-arm phase II basket trial for advanced tumors selected according to predefined molecular profiles (NCT03767075). Overall, $36 \%$ of the patients of the CCE cohort presented here were recommended for one of the BoB treatment arms available at the moment of the molecular tumor board discussion (Figure 2A). These were mostly associated with the use of immune checkpoint inhibitors in the presence of putative loss-of-function events in DNA damage repair genes, as estimated by the MTBP variant interpretation. However, the majority $(60 \%)$ of these cases were not finally enrolled in the BoB trial, 
mostly due to the patient's clinical deterioration or subsequent screening failures. This further emphasizes the importance of deploying systems that can support an efficient and rapid trial recruitment at the point of clinical decision-making.

The MTBP retrieves the trials' eligibility criteria from in-house databases gathering up-to-date clinical, pathologic and molecular requisites. These requisites are stated following an ad-hoc syntax adapted to the growing complexity of cancer biomarkers, which are defined by the presence (or absence) of a given combination of genomic alterations and/or genomic signatures. Trials database also includes prioritization rules in case that the allocation to multiple trials is possible, as well as variant interpretation nuances to be used by the MTBP analysis. One example of the latter is the evidence required for considering variants in a given actionable gene as functionally relevant; in general, we only match clinical trials with tumor variants whose effect is based on well-curated studies or bona fide biological assumptions, but lower evidence such as bioinformatic predictions are also considered for emerging biomarkers associated with less characterized genes. Upfront agreement on these details enables the MTBP to refine the actionability flags issued in the reports, which facilitates efficient discussions during the molecular tumor board meetings and increases the consistency of the clinical decision-making.

Importantly, the MTBP actionability flags can be used to automate the detection of other events of potential clinical relevance. For example, and in collaboration with the CCE genetic counseling task force, we have established unified criteria to flag germline variants requiring genetic specialist referral (Appendix 1). As a result, the MTBP issued genetic counseling alerts for 49 germline variants in 48 individuals ( $57 \pm 13$ years), which represent $13 \%$ of the CCE cohort with paired tumor/normal samples sequencing available (with a cancer type distribution similar to that of the overall cohort, see Table 1). Of note, three $(6 \%)$ of these variants showed a low variant allele frequency in the tumor sample, and thus they would have been not contemplated as of potential germline origin with tumor-only sequencing data, as per published criteria24. Genetic counselor review deemed these findings as of clinical significance in all the cases, although close to half $(44 \%)$ of the variant carriers did not meet personal criteria for clinical germline testing25,26 (Figure 2B). Moreover, a considerable (18\%) proportion of these pathogenic germline variants were found in genes not associated with the patient's index cancer, which further complicates their discovery via standard guidelines-directed genetic testing. Overall, these results illustrate the importance of the oncology setting for screening hereditary cancer susceptibility variants27, and the value of the MTBP to streamline that task.

\section{The MTBP technology}

The MTBP provides a single unified framework for sharing and harnessing NGS data across CCE centers. De-identified patient clinical and pathological information is fetched from a centralized electronic case report forms system, while sequencing data files are retrieved from different institutional and external laboratories. Data transfer, storage and access is implemented by a set of technical measures in accordance with the European legal framework under compliance with data protection regulation. After data capture, the system triggers a number of pipelines for data integrity control, format harmonization 
and variant interpretation as appropriate. This ultimately creates the corresponding patient reports, which are immediately shared with the clinical investigators. The whole process is fully automated and thus performed in a negligible amount of time, which dramatically reduces the efforts required for case preparation. At present, our turnaround time from biopsy collection to sequencing report availability is less than 14 days, as required in patients whose clinical condition can rapidly deteriorate28.

The MTBP patient reports are HTML web-based documents accessible for the clinical investigators via a secure online platform2. These reports are discussed in weekly virtual molecular tumor board meetings, in which members of each CCE center connect from different locations and agree on clinical recommendations. As discussed before, genomic alterations are flagged in the MTBP report according to predefined expert actionability criteria, and all the results appear systematically organized in a userfriendly, readily interpretable view (Figure 2B). In addition, further information and variant annotation details are accessible via interactive elements of the HTML report, which empowers an in-depth revision of the content and supporting evidence. Although the MTBP can also distribute summarized reports in PDF format, we believe that working with interactive data-rich documents is more appropriate in the context of academic medical centers, in which molecular tumor boards discuss more complex cases and serve as a venue for continued education in genomics-driven oncology. Of note, we observed a learning curve to use the MTBP system lasting for approximately 25 patients (Figure $2 \mathrm{C}$ ). After that, the amount of time devoted to discussing each case averaged less than three minutes, which is key for scaling the process to a large number of patients.

At the moment of writing this manuscript, the MTBP supports the interpretation of genomics data and has recently incorporated gene expression analysis. As one of the founding principles of CCE is to share new tools with the cancer research community, we have created an open version of the MTBP analytical framework available at http://mtbp.org (Supp Figure 4). This public resource supports a general interpretation of the gene variants uploaded by the user, which (although more narrowed than that deployed for CCE projects) may be of interest for researchers outside of our network. In order to support innovative clinical trials for next generation precision medicine, we are currently working on the incorporation of emerging technologies such as proteomics, ex-vivo drug screening and digital pathology into the MTBP. Ultimately, we envision the platform as a catalyzer for systems-based precision oncology strategies capable of integrating multiple levels of molecular and imaging data and inform treatment decisions throughout the patient's disease course.

\section{Discussion}

The MTBP implements an efficient process to support the biomarker-driven clinical decision-making at scale and creates a unified platform for developing new trials in a truly collaborative manner. As the complexity of cancer biomarkers continues to grow, automating the interpretation and reporting of sequencing results decreases the need for manual procedures and facilitates rapid, comprehensive and consistent clinical decision-making. In addition, the MTBP creates the infrastructure to systematically gather the molecular and clinical information in a "bio-repository" of data, which supports the discovery 
of new cancer biomarkers and insights for future trial designs. However, deploying the MTBP across the CCE network raised multiple challenges beyond the development of the platform itself, such as: (i) ensuring the interoperability with the information technology systems of each connected center; (ii) automating the retrieval of clinical and sequencing data provided by different facilities; (iii) developing user-friendly interfaces for distinct user types, such as medical practitioners, project managers and data analysts; (iv) coordinating the efforts to agree on variant interpretation criteria and actionability prioritization; and ( $v$ ) creating the associated resources, such as a database with up-to-date information of the clinical trials open for recruitment across the network. These tasks require expertise from domains such as medical software regulation, cyber-security and front-end development, which is not easily available in the academic setting and thus creates needs for collaboration with industry partners. In conclusion, we believe that streamlining digital systems like the MTBP at the point of care is key to address current challenges of biomarker-driven precision oncology, but the success of these initiatives relies on the long-term investment needed to develop and maintain the technology.

\section{Declarations}

\section{Acknowledgements}

We acknowledge the Ensembl team at EMBL-EBI and Wanding Zhou for the development and support of the Ensembl Variant Effect Predictor and TransVar tools, respectively, the IT department at SciLifeLab for their technical support in deploying the portal, and the developers of publicly available genomic knowledgebases for their work in curating these resources. The project has received support from the Personalized Cancer Medicine program at Karolinska funded by The Cancer Research Funds of Radiumhemmet, Swedish Foundation of Strategic Research (PI Lehtiö), Stockholm County Council (ALFfunding, PI Lehtiö) and from Cancer Research UK in the Cambridge Centre. We also acknowledge F. Hoffmann-la Roche Ltd. for their support in the Basket of Baskets trial (NCT03767075).

\section{Competing interests:}

David Tamborero reports receiving honoraria for speaker activities and advisory role for Roche. Rodrigo Dienstmann reports receiving honoraria for speaker activities from Roche, Ipsen, Amgen, Sanofi, Servier Laboratories, Merck Sharp \& Dohme; advisory role from Roche and Boehringer Ingelheim; and research grants from Merck and Pierre Fabre. Irene Braña reports consultant or advisory role for Orion Pharma; speaker activities for BMS; travel grants from AstraZeneca and Merck Serono; principal investigator of clinical trials for AstraZeneca, BMS, Celgene, Gliknik, GSK, Janssen, KURA, MSD, Novartis, Orion Pharma, Pfizer, Shattuck, Northern Biologics, Rakutan Aspirian and Nanobiotics. Richard Baird reports consultant or advisory roles (with funding to institution) for AstraZeneca, Daiichi-Sankyo, Lilly, Molecular Partners, Novartis, Roche, Shionogi; principal/sub-Investigator of clinical trials for Astex, AstraZeneca, BoehringerIngelheim, Boston Therapeutics, Genentech/Roche, Johnson\&Johnson, Lilly, Molecular Partners, PharmaMar, Roche, Sanofi-Aventis, Shionogi and Taiho; and research grants from AstraZeneca, Boehringer-Ingelheim and Genentech. Christophe Massard reports consultant/advisory fees from Amgen, 
Astellas, Astra Zeneca, Bayer, BeiGene, BMS, Celgene, Debiopharm, Genentech, Ipsen, Janssen, Lilly, Medlmmune, MSD, Novartis, Pfizer, Roche, Sanofi, Orion; principal/sub-Investigator of clinical trials for Abbvie, Aduro, Agios, Amgen, Argen-x, Astex, AstraZeneca, Aveopharmaceuticals, Bayer, Beigene, Blueprint, BMS, Boeringer Ingelheim, Celgene, Chugai, Clovis, Daiichi Sankyo, Debiopharm, Eisai, Eos, Exelixis, Forma, Gamamabs, Genentech, Gortec, GSK, H3 biomedicine, Incyte, Innate Pharma, Janssen, Kura Oncology, Kyowa, Lilly, Loxo, Lysarc, Lytix Biopharma, Medimmune, Menarini, Merus, MSD, Nanobiotix, Nektar Therapeutics, Novartis, Octimet, Oncoethix, Oncopeptides AB, Orion, Pfizer, Pharmamar, Pierre Fabre, Roche, Sanofi, Servier, Sierra Oncology, Taiho, Takeda, Tesaro and Xencor. Richard Schlenk reports research funding from Pfizer, AstraZeneca, PharmaMar, Roche, Daiichi Sankyo; speakers honoraria from Pfizer, Daiichi Sankyo, Novartis; participation in Ad Boards: Pfizer, Daiichi Sankyo, Novartis. Giovanni Apolone reports no conflicts of interests with regards to the topic at hand; as scientific director of the Fondazione IRCCS Istituto Nazionale dei Tumori he is legally responsible for contracts with Pharma and other funding agencies. Carlos Caldas is a member of the External Science Panel of AstraZeneca, a member of Illumina's Scientific Advisory Board and his laboratory has received research grants (administered by the University of Cambridge) from Genentech, Roche, AstraZeneca, and Servier. Stefan Fröhling reports a consulting or advisory role, having received honoraria, research funding, and/or travel/accommodation expenses funding from the following for-profit companies: Bayer, Roche, Amgen, Eli Lilly, PharmaMar, AstraZeneca, and Pfizer. Elena Garralda reports consultant honoraria from Roche/Genentech, F.Hoffmann/La Roche, Ellipses Pharma, Neomed Therapeutics Inc, Boehringer Ingelheim - Janssen Global Services, AstraZeneca, SeaGen, TFS - Alkermes; research for Novartis / Roche; principal/sub-Investigator of Clinical Trials for Principia Biopharma Inc., Lilly, S.A, Novartis Farmacéutica, S.A, Genentech Inc, Loxo Oncology Inc, F.Hoffmann La Roche Ltd, Symphogen A/S, Merck, Sharp \& Dohme de España, S.A, Incyte Biosciences International, Pharma Mar, S.A.U, Kura Oncology Inc, Macrogenics Inc, Glycotope Gmbh, Pierre Fabre Medicament, Cellestia Biotech, Menarini Ricerche Spa, Blueprint Medicines Corporation, Beigene Usa, Inc, Sierra Oncology, Inc, Genmab B.V; travel grants from Bristol-Mayers Squibb, Merck Sharp \& Dohme, Menarini, Glycotope; speakers bureau for Bristol-Mayers Squibb, Merck Sharp \& Dohme, Roche, ThermoFisher. Josep Tabernero reports personal financial interest in form of scientific consultancy role for Array Biopharma, AstraZeneca, Bayer, BeiGene, Boehringer Ingelheim, Chugai, Genentech, Inc., Genmab A/S, Halozyme, Imugene Limited, Inflection Biosciences Limited, Ipsen, Kura Oncology, Lilly, MSD, Menarini, Merck Serono, Merrimack, Merus, Molecular Partners, Novartis, Peptomyc, Pfizer, Pharmacyclics, ProteoDesign SL, Rafael Pharmaceuticals, F. Hoffmann-La Roche Ltd, Sanofi, SeaGen, Seattle Genetics, Servier, Symphogen, Taiho, VCN Biosciences, Biocartis, Foundation Medicine, HalioDX SAS and Roche Diagnostics. Emile Voest reports no conflict of interest with regards to the topic at hand; as medical director of the Netherlands Cancer Institute he is legally responsible for all contracts with pharma. Jordi Rodon reports research funding from Bayer \& Novartis; clinical research for Spectrum Pharmaceuticals, Tocagen, Symphogen, BioAlta, Pfizer, GenMab, CytomX, KELUN-BIOTECH, Takeda-Millennium, GLAXOSMITHKLINE, IPSEN; scientific advisory board for Novartis, Eli Lilly, Orion Pharmaceuticals, Servier Pharmaceuticals, Peptomyc, Merck Sharp \& Dohme, Kelun Pharmaceuticals/Klus Pharma, Spectrum Pharmaceuticals Inc., Pfizer, Roche Pharmaceuticals, Ellipses 
Pharma; research funding for Bayer \& Novartis. Janne Lehtiö reports research funding from AstraZeneca, Novartis and GE healthcare, and is co-founder and shareholder of FenoMark Diagnostics Ab.

\section{Other statements}

This manuscript describes a consecutive cohort of advanced tumor patients pre-registered in the Basket of Baskets (NCT03767075) trial during a two years period (no data have been excluded from the results presented here). This manuscript describes clinical and genomic findings aggregated at the cohort level and are all available in the manuscript text/figures. Raw sequencing data may be distributed upon request. The Vall d'Hebron Institute of Oncology (VHIO) is the sponsor of the Basket of Baskets trial. The protocol was submitted through the Voluntary Harmonization Procedure (VHP) and approved by the Medicines \& Healthcare products Regulatory Agency (MHRA) in the UK. Subsequently, the competent authorities in Spain (Agencia Española de Medicamentos y Productos Sanitarios - AEMPS), France (Agence nationale de sécurité du medicament et des produits de santé - ANSM), Germany (Bundesinstitut für Impfstoffe und biomedizinische Arzneimittel), The Netherlands (Centrale Commissie Mensgebonden Onderzoek - CCMO), and Sweden (Läkemedelsverket) provided local approval. Ethics Committee approvals have been obtained in Spain, UK, France, The Netherlands and Sweden, under the EduraCT project number 2018-005108-89. All patients signed an informed consent form for the pre-registration, and another for the trial in the case of recruitment. The clinical and sequencing data transfer, storage and access complies with legal and ethical regulations as appropriate.

\section{References}

1. Eggermont AMM, Apolone G, Baumann M, et al. Cancer Core Europe: A translational research infrastructure for a European mission on cancer. Mol Oncol. 2019;13(3):521-527. doi:10.1002/18780261.12447

2. Tamborero $\mathrm{D}$, Dienstmann $\mathrm{R}$, Rachid $\mathrm{MH}$, et al. Support systems to guide clinical decision-making in precision oncology: The Cancer Core Europe Molecular Tumor Board Portal. Nat Med. 2020;26(7):992-994. doi:10.1038/s41591-020-0969-2

3. Tavtigian SV, Greenblatt MS, Harrison SM, et al. Modeling the ACMG/AMP Variant Classification Guidelines as a Bayesian Classification Framework. Genet Med Off J Am Coll Med Genet. 2018;20(9):1054-1060. doi:10.1038/gim.2017.210

4. Landrum MJ, Lee JM, Benson M, et al. ClinVar: improving access to variant interpretations and supporting evidence. Nucleic Acids Res. 2018;46(D1):D1062-D1067. doi:10.1093/nar/gkx1153

5. Cline MS, Liao RG, Parsons MT, et al. BRCA Challenge: BRCA Exchange as a global resource for variants in BRCA1 and BRCA2. PLoS Genet. 2018;14(12). doi:10.1371/journal.pgen.1007752

6. Chakravarty D, Gao J, Phillips S, et al. OncoKB: A Precision Oncology Knowledge Base. JCO Precis Oncol. 2017;(1):1-16. doi:10.1200/P0.17.00011

7. Griffith M, Spies NC, Krysiak K, et al. CIViC is a community knowledgebase for expert crowdsourcing the clinical interpretation of variants in cancer. Nat Genet. 2017;49(2):170-174. doi:10.1038/ng.3774 
8. Tamborero D, Rubio-Perez C, Deu-Pons J, et al. Cancer Genome Interpreter annotates the biological and clinical relevance of tumor alterations. Genome Med. 2018;10(1):25. doi:10.1186/s13073-0180531-8

9. The 1000 Genomes Project Consortium. A global reference for human genetic variation. Nature. 2015;526(7571):68-74. doi:10.1038/nature15393

10. Karczewski KJ, Francioli LC, Tiao G, et al. The mutational constraint spectrum quantified from variation in 141,456 humans. Nature. 2020;581(7809):434-443. doi:10.1038/s41586-020-2308-7

11. Richards S, Aziz N, Bale S, et al. Standards and guidelines for the interpretation of sequence variants: a joint consensus recommendation of the American College of Medical Genetics and Genomics and the Association for Molecular Pathology. Genet Med Off J Am Coll Med Genet. 2015;17(5):405-424. doi:10.1038/gim.2015.30

12. Abou Tayoun AN, Pesaran T, DiStefano MT, et al. Recommendations for interpreting the loss of function PVS1 ACMG/AMP variant criterion. Hum Mutat. 2018;39(11):1517-1524. doi:10.1002/humu.23626

13. Tamborero D, Gonzalez-Perez A, Lopez-Bigas N. OncodriveCLUST: exploiting the positional clustering of somatic mutations to identify cancer genes. Bioinforma Oxf Engl. 2013;29(18):2238-2244. doi:10.1093/bioinformatics/btt395

14. Chang MT, Asthana S, Gao SP, et al. Identifying recurrent mutations in cancer reveals widespread lineage diversity and mutational specificity. Nat Biotechnol. 2016;34(2):155-163. doi:10.1038/nbt.3391

15. Gao J, Chang MT, Johnsen HC, et al. 3D clusters of somatic mutations in cancer reveal numerous rare mutations as functional targets. Genome Med. 2017;9(1):4. doi:10.1186/s13073-016-0393-x

16. Kircher M, Witten DM, Jain P, O’Roak BJ, Cooper GM, Shendure J. A general framework for estimating the relative pathogenicity of human genetic variants. Nat Genet. 2014;46(3):310-315. doi:10.1038/ng.2892

17. Zhou W, Chen T, Chong Z, et al. TransVar: a multilevel variant annotator for precision genomics. Nat Methods. 2015;12(11):1002-1003. doi:10.1038/nmeth.3622

18. McLaren W, Gil L, Hunt SE, et al. The Ensembl Variant Effect Predictor. Genome Biol. 2016;17(1):122. doi:10.1186/s13059-016-0974-4

19. Rivera-Muñoz EA, Milko LV, Harrison SM, et al. ClinGen Variant Curation Expert Panel experiences and standardized processes for disease and gene-level specification of the ACMG/AMP guidelines for sequence variant interpretation. Hum Mutat. 2018;39(11):1614-1622.

doi:https://doi.org/10.1002/humu.23645

20. Wagner $A H$, Walsh B, Mayfield G, et al. A harmonized meta-knowledgebase of clinical interpretations of somatic genomic variants in cancer. Nat Genet. 2020;52(4):448-457. doi:10.1038/s41588-0200603-8

21. Wagner AH, Babb L, Alterovitz G, et al. The GA4GH Variation Representation Specification (VRS): a Computational Framework for the Precise Representation and Federated Identification of Molecular 
Variation. bioRxiv. Published online January 17, 2021:2021.01.15.426843.

doi:10.1101/2021.01.15.426843

22. Mateo J, Chakravarty D, Dienstmann $\mathrm{R}$, et al. A framework to rank genomic alterations as targets for cancer precision medicine: the ESMO Scale for Clinical Actionability of molecular Targets (ESCAT). Ann Oncol Off J Eur Soc Med Oncol. 2018;29(9):1895-1902. doi:10.1093/annonc/mdy263

23. Li MM, Datto M, Duncavage EJ, et al. Standards and Guidelines for the Interpretation and Reporting of Sequence Variants in Cancer: A Joint Consensus Recommendation of the Association for Molecular Pathology, American Society of Clinical Oncology, and College of American Pathologists. J Mol Diagn JMD. 2017;19(1):4-23. doi:10.1016/j.jmoldx.2016.10.002

24. Mandelker D, Donoghue M, Talukdar S, et al. Germline-focussed analysis of tumour-only sequencing: recommendations from the ESMO Precision Medicine Working Group. Ann Oncol. 2019;30(8):12211231. doi:10.1093/annonc/mdz136

25. Paluch-Shimon S, Cardoso F, Sessa C, et al. Prevention and screening in BRCA mutation carriers and other breast/ovarian hereditary cancer syndromes: ESMO Clinical Practice Guidelines for cancer prevention and screening. Ann Oncol Off J Eur Soc Med Oncol. 2016;27(suppl 5):v103-v110. doi:10.1093/annonc/mdw327

26. Stjepanovic N, Moreira L, Carneiro F, et al. Hereditary gastrointestinal cancers: ESMO Clinical Practice Guidelines for diagnosis, treatment and follow-upt. Ann Oncol Off J Eur Soc Med Oncol. 2019;30(10):1558-1571. doi:10.1093/annonc/mdz233

27. Assessment of Clinical Benefit of Integrative Genomic Profiling in Advanced Solid Tumors | Genetics and Genomics | JAMA Oncology I JAMA Network. Accessed March 19, 2021. https://jamanetwork.com/journals/jamaoncology/fullarticle/2776760

28. Chen A, Flaherty K, O'Dwyer PJ, et al. Tumor Genomic Profiling Practices and Perceptions: A Survey of Physicians Participating in the NCI-MATCH Trial. JCO Precis Oncol. 2020;(4):1207-1216. doi:10.1200/PO.20.00217

\section{Tables}

Table 1. Characteristics of the 500 tumors of the Cancer Core Europe prospective cohort 


\begin{tabular}{|c|c|}
\hline Age, median (IQR) & $59 y(49-67)$ \\
\hline Female gender, n (\%) & $300(60 \%)$ \\
\hline Paired samples ${ }^{\mathrm{a}}, \mathrm{n}(\%)$ & $354(70 \%)$ \\
\hline Primary tumor sample ${ }^{\mathrm{b}}, \mathrm{n}(\%)$ & $270(54 \%)$ \\
\hline Tumor purityc ${ }^{\mathrm{C}}$ median (IQR) & $60 \%(35-80)$ \\
\hline Primary Cancer type, n (\%) & \\
\hline Breast carcinoma & $100(20 \%)$ \\
\hline Colorectal adenocarcinoma & $65(13 \%)$ \\
\hline Ovarian epithelial tumor & $54(11 \%)$ \\
\hline Esophagogastric adenocarcinoma & $30(6 \%)$ \\
\hline Cholangiocarcinoma & $25(5 \%)$ \\
\hline Pancreatic adenocarcinoma & $20(4 \%)$ \\
\hline Cancer of unknown primary & $15(3 \%)$ \\
\hline Prostate adenocarcinoma & $10(2 \%)$ \\
\hline Salivary carcinoma & $10(2 \%)$ \\
\hline Pleural mesothelioma & $10(2 \%)$ \\
\hline Gallbladder cancer & $10(2 \%)$ \\
\hline Others & $150(30 \%)$ \\
\hline
\end{tabular}

Patients with advanced/refractory disease considered for Cancer Core Europe clinical trials from January 2019 to January 2021. Note that the cohort is biased towards those cancer types that were more suited to the clinical trials opened during that time period. All the samples were profiled by targeted next-generation sequencing panels (from 326 to 350 cancer genes evaluated, depending on the assay).

a Sequencing of paired white blood cells and tumor tissue samples identifying germline versus tumor somatic variants; tumor sample only sequenced otherwise

${ }^{\mathrm{b}}$ Sequenced tumor sample obtained from the primary tumor; sample from a metastatic site otherwise

${ }^{\mathrm{c}}$ Percentage of tumor content in the tumor sample as reported by pathology assessment

\section{Figures}


A Cancer gene variant interpretation

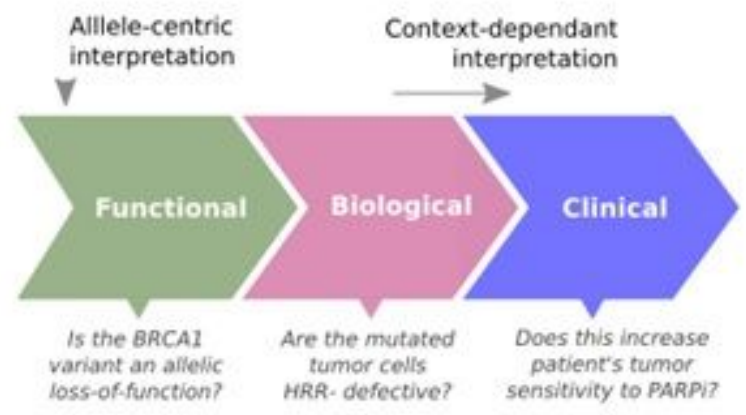

B Functional classification of tumor mutations (CCE cohort)

Accumulated variants Unique variants $(n=9,194) \quad(n=6,748)$
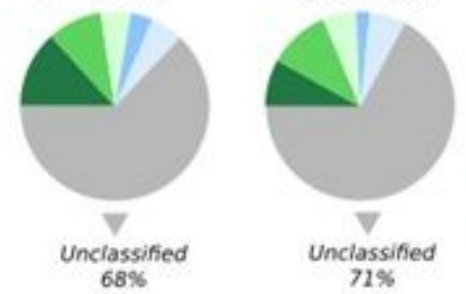

Functionally relevant evidence:

Curated

effect

assumption

$\square$ Bioinformatics.

metrics

Functionally neutral evidence:

Curated effect $\square$ Bioinformatics.

\section{Cunctional classification of tumor mutations - per gene (CCE cohort)}

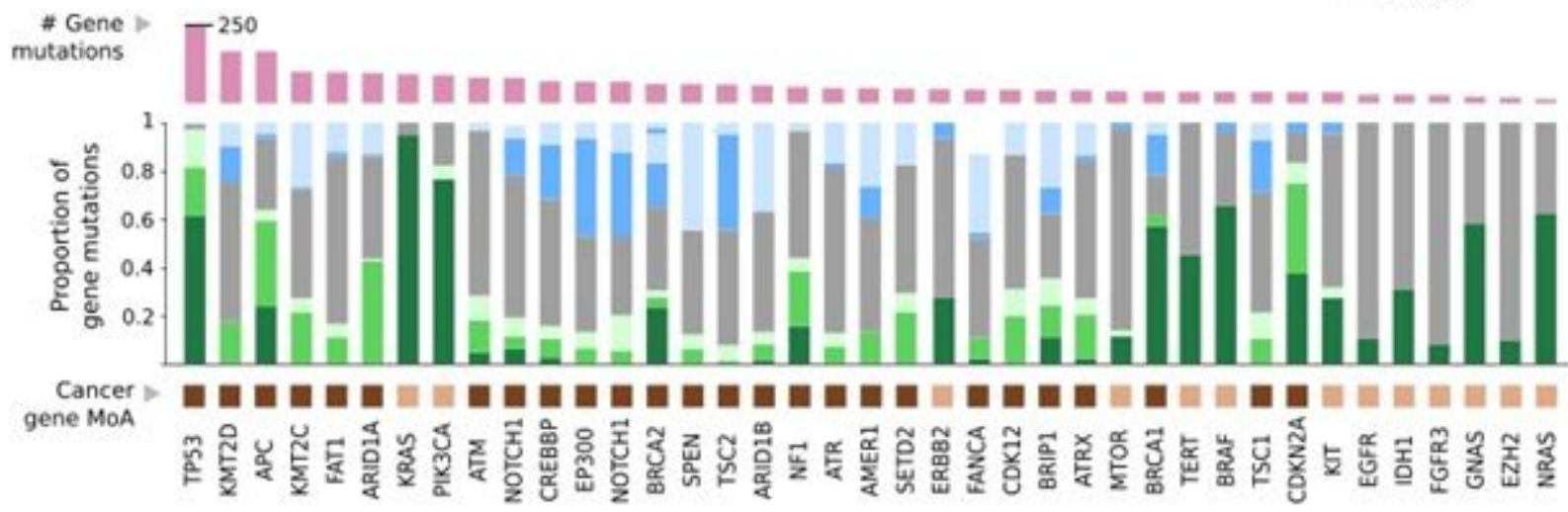

\section{Tumors with mutations matching reported drug sensitivity biomarkers (CCE cohort)}

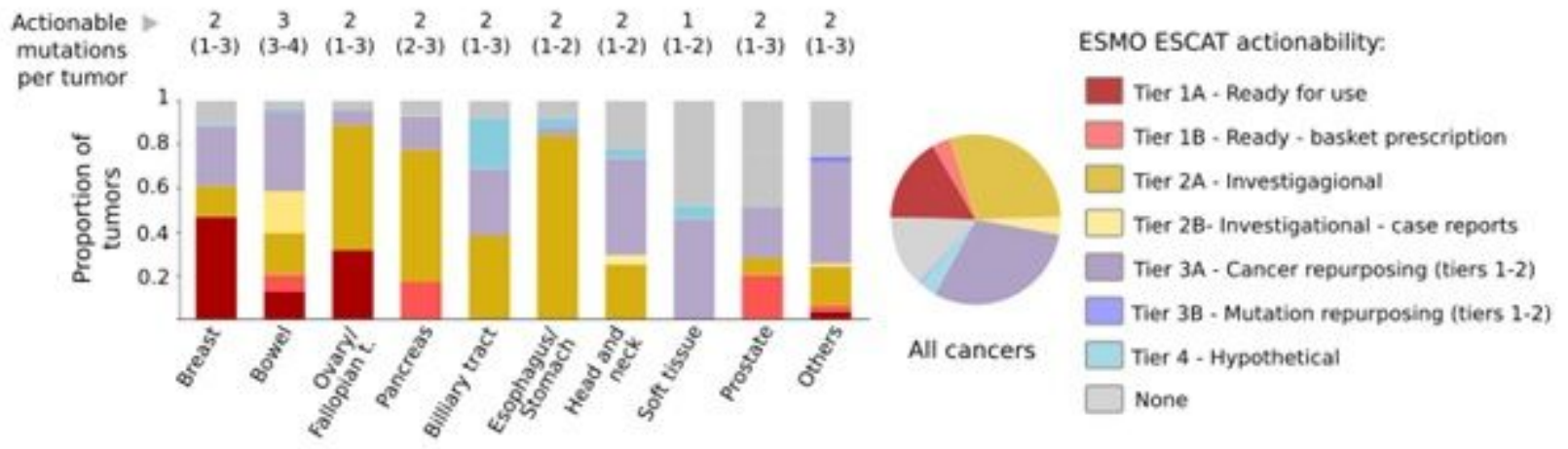

\section{Figure 1}

Panel A: Representation of distinct 'phenotype resolutions' for cancer gene variant interpretation. The functional relevance evaluates the allele-centric effect of a given genomic variant in a tumor contextagnostic manner, while the interpretation of its relevance for biological traits and clinical outcomes factors in additional considerations (such as germline versus tumor somatic origin, co-occurring alterations in the same or other genes and/or patient's cancer type). Specific questions that are addressed in each step are exemplified below the graph for a given BRCA1 mutation (HRR=homologous recombination repair, PARPi=Poly-ADP ribose polymerase inhibitors). Panel B: Functional classification for the tumor variants (single nucleotide changes and small insertions/deletions) observed in the Cancer 
Core Europe (CCE) cohort, as profiled by cancer genes panel sequencing. Results are shown according to each source of evidence employed by the MTBP interpretation pipeline after removing all the variants considered as neutral by bona fide assumptions (such as common polymorphisms and variants that do not alter the protein sequence). Left pie chart represents overall counts of variants, while the right pie chart represents counts of unique variants. Panel C: Results as in panel B detailed for several of the most recurrently mutated cancer genes in the CCE cohort (MoA=mechanism of action, representing genes that (predominantly) act as tumor suppressors (dark brown) or oncogenes (light brown)). Most of the tumor suppressors bear a variety of variants with null consequence types (such as frameshift or nonsense mutations) whose classification mostly relies on the use of bona fide biological assumptions. Exceptions are tumor suppressors whose variants are largely characterized due to ongoing efforts (such as BRCA1 and BRCA2) and/or enriched by specific variants driving dominant negative effects (such as TP53 and -to a lesser extent- PTEN). On the other hand, some oncogenes are enriched by specific variants with wellknown gain-of-function effects (such as KRAS and BRAF), while others bear a variety of variants that remain mostly unclassified (such as ERBB2 and FGFR3). Panel D: Tumor (or germline, when appropriate) variants observed across the CCE cohort and matching with biomarkers of drug response reported at the moment of writing this manuscript. Only biomarkers of drug sensitivity (not resistance or toxicity) are displayed here. Color bars represent the highest ESMO-ESCAT level of actionability of all the biomarkers matched in each tumor, after grouping the samples by their tissue of origin. Numbers above the bars represent the median (percentile 25-75) number of tumor variants matching drug biomarkers regardless of their level of actionability. Of note, biomarkers defined by functional criteria (such as 'BRCA1/2 loss-offunction and olaparib response') are matched here with tumor mutations classified as functionally relevant according to any of the supporting evidence employed by the MTBP pipeline. 
A

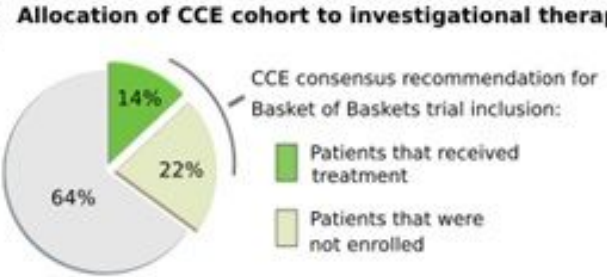

B Genetic counseling alerts in the CCE cohort

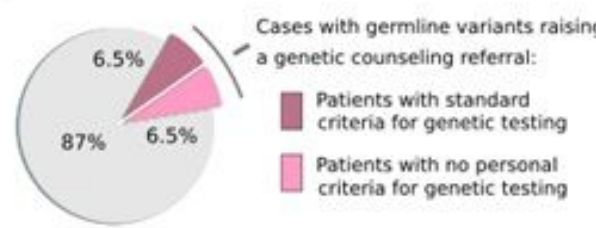

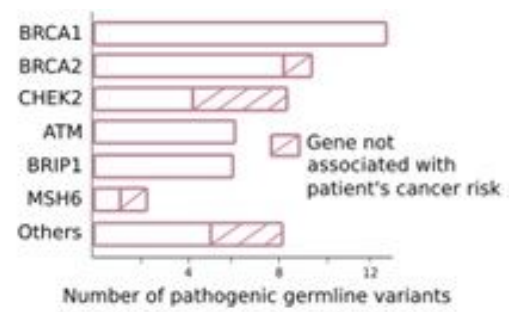
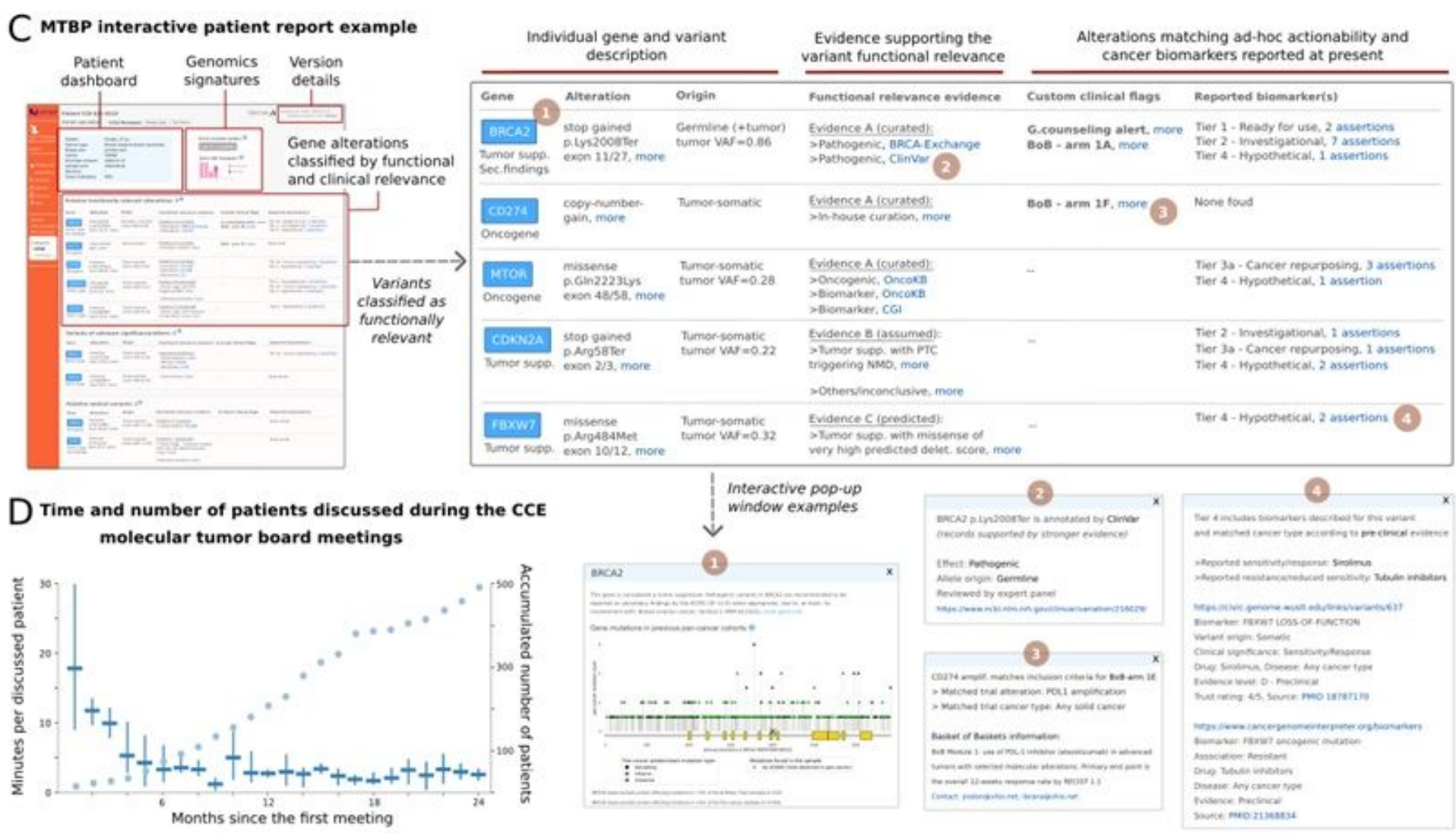

\section{Figure 2}

Panel A: Patient cases in which the CCE molecular tumor board recommended the allocation to one of the Basket of Baskets trial arms opened at the moment of the manuscript writing. The pie chart details the proportion of these patients that were not finally enrolled due to clinical deterioration and/or screening failures. For the remaining cases, possibilities for other available clinical trials or compassionate drug prescriptions were decided in each patient's medical institution without requiring CCE consensus (data not shown). Panel B: Patient cases in which the MTBP issued an alert due to germline variant(s) potentially associated with inherited cancer risk (only the subset of 375 patients with normal and tumorpaired samples sequencing available are included here). The pie chart details the proportion of these cases that would have been missed with a germline testing approach based on current clinical guidelines, as variant carriers did not meet personal criteria for doing so. Bar plots represent the genes bearing these germline variants, which in all cases were deemed as actionable by genetic specialist review. Striped bars detail cases in which the pathogenic variant was found in a gene not currently established as increasing the risk for the patient's index cancer type. Panel C: The MTBP patient reports provide an integrated dashboard with de-identified clinical and pathology information, as retrieved from the corresponding electronic case report form, together with sequencing assay details and version control of the resources 
employed to annotate the results. Relevant molecular signatures (such as tumor mutation burden) are listed together with individual gene alterations, which appear organized in three different tables according to their functional classification (functionally relevant, unclassified and functionally neutral). These tables provide, among others, the evidence supporting such functional classification and the associated clinical actions, including in-house initiatives (e.g. clinical trials eligibility and genetic counseling alerts) and matching with cancer biomarkers reported to date (diagnosis, prognosis and drug response, tiered according to the ESMO-ESCAT scale of actionability). Additional information and annotation details can be accessed by the use of interactive elements in the HTML report, as exemplified here by the pop-up windows opened in numbers 1-4. Panel D: The lines represent the time (median and interquartile range) devoted to discussing each patient's case during the CCE virtual molecular tumor board meetings held during the initial 24 months period. The circles represent the accumulated number of reviewed cases.

\section{Supplementary Files}

This is a list of supplementary files associated with this preprint. Click to download.

- Supplementarylnformation.pdf 